\title{
Priority setting for the implementation of artemisinin-based combination therapy policy in Tanzania: evaluation against the accountability for reasonableness framework
}

\author{
Amani Thomas Mori ${ }^{1,2^{*}}$ and Eliangiringa Amos Kaale ${ }^{3}$
}

\begin{abstract}
Background: Priority setting for artemisinin-based antimalarial drugs has become an integral part of malaria treatment policy change in malaria-endemic countries. Although these drugs are more efficacious, they are also more costly than the failing drugs. When Tanzania changed its National Malaria Treatment Policy in 2006, priority setting was an inevitable challenge. Artemether-lumefantrine was prioritised as the first-line drug for the management of uncomplicated malaria to be available at a subsidized price at public and faith-based healthcare facilities.

Methods: This paper describes the priority-setting process, which involved the selection of a new first-line antimalarial drug in the implementation of artemisinin-based combination therapy policy. These descriptions were further evaluated against the four conditions of the accountability for reasonableness framework. According to this framework, fair decisions must satisfy a set of publicity, relevance, appeals, and revision and enforcement conditions.

In-depth interviews were held with key informants using pretested interview guides, supplemented with a review of the treatment guideline. Purposeful sampling was used in order to explore the perceptions of people with different backgrounds and perspectives. The analysis followed an editing organising style.

Results: Publicity: The selection decision of artemether-lumefantrine but not the rationale behind it was publicised through radio, television, and newspaper channels in the national language, Swahili. Relevance: The decision was grounded on evidences of clinical efficacy, safety, affordability, and formulation profile. Stakeholders were not adequately involved. There was neither an appeals mechanism to challenge the decision nor enforcement mechanisms to guarantee fairness of the decision outcomes.
\end{abstract}

Conclusions: The priority-setting decision to use artemether-lumefantrine as the first-line antimalarial drug failed to satisfy the four conditions of the accountability for reasonableness framework. In our understanding, this is the first study to evaluate priority-setting decisions for new drugs in Tanzania against the accountability for reasonableness framework. In addition to the demand for enhanced stakeholder involvement, publicity, and transparency, the study also calls for the institution of formal appeals, revision, and regulatory mechanisms in the future change of malaria treatment policies.

\footnotetext{
* Correspondence: pax_amani@yahoo.com

'Department of Pharmaceutics and Pharmacy Practice, School of Pharmacy,

Muhimbili University of Health and Allied Sciences, Dar es Salaam, Tanzania

Full list of author information is available at the end of the article
} 


\section{Background}

Malaria case management with pharmaceuticals is a major challenge in many malaria-endemic countries. Old but cheap and effective medicines are increasingly facing resistance. By 2009, nearly all Plasmodium falciparum malaria-endemic countries, most being in subSaharan Africa, had changed their treatment policies to artemisinin-based combination therapies $[1,2]$. On the other hand, rapidly increasing pharmaceutical expenditure poses another major obstacle and has led to an increased need for prioritisation in pharmaceuticals worldwide $[3,4]$. When Tanzania changed its National Malaria Treatment Policy in 2006, three choices of artemisinin-based combination therapies recommended by the World Health Organisation (WHO) were available [5]. The guiding principle was to guarantee access to antimalarial drugs that are safe, affordable, effective, acceptable, and of good quality for patients infected with malaria [6]. Limited resources, especially for publicly funded healthcare systems, mean that priorities must be set, since not all the available interventions can be provided for all who need them [7]. Among the available alternatives, artemether-lumefantrine (ALu) was prioritised to be the first-line drug for the management of uncomplicated malaria to be available at a subsidized price in both public and faith-based healthcare facilities.

Tanzania has a fairly well-distributed healthcare system, within which $66 \%$ of all the healthcare facilities are owned by the government [8]. About $80 \%$ of the population has access to healthcare services that, to a large extent, do not meet the minimum acceptable standards [9]. In 1993, the government introduced a cost-sharing policy and health insurance schemes to complement government financing and as an attempt to improve the quality of services [10]. Currently, about $15 \%$ of the population is covered by health insurance [11]. Households contribute $47 \%$ of healthcare financing through out-of-pocket expenditure and $43 \%$ is financed by the government and donor support [12].
Rational malaria treatment policy formulation presents challenges to many endemic countries. At times, donors may favour an approach that conflicts with the national preferences $[13,14]$. Studies on the previous policy change in Tanzania, from chloroquine to sulphadoxinepyrimethamine, described the process as irrational. It was characterised by disagreements among the stakeholders, mistrust of evidence, and accusations of influence from pharmaceutical companies $[15,16]$. The change of policy was influenced by only a few individuals who were involved in various ways with malaria [16].

\section{Accountability for reasonableness and its applications in Tanzania}

The accountability for reasonableness framework (AFR) (Table 1) is a procedural framework that was developed as an approach to guarantee fairness and legitimacy of priority-setting decisions in health [17-19]. When properly implemented, AFR can prevent the development of fairness and legitimacy problems grounded on mistrust of the processes. The framework outlines a roadmap of four key conditions that must be fully fulfilled in the decision-making process. In recent years, despite its limited application in developing countries, AFR has been gaining popularity in Tanzania, especially at the district level [20-22].

District and regional health planners have applauded the framework for its ability to enhance participation, transparency, and the development of relevant criteria for decision making [20]. Where it has been used to evaluate priority-setting decisions, it facilitated the identification of key areas that needed improvement [21,22]. Daniels and Sabin, who developed the framework, suggest that 'accountability for reasonableness' is relevant to pharmaceutical priority setting:

Managing access to pharmaceuticals is a microcosm of the limit-setting problems of healthcare systems as a whole. If we are right that accountability for reasonableness is a solution to the legitimacy problem in health

Table 1 Accountability for reasonableness framework

\begin{tabular}{ll}
\hline Condition & Description \\
\hline Publicity & $\begin{array}{l}\text { Decisions regarding both direct and indirect limits to care and their rationales must be publicly accessible. The process of priority } \\
\text { setting must be open and transparent and consultations and public hearings should be held. Publicity and involvement of key } \\
\text { stakeholders are particularly important in contexts where policy and programmatic decisions occur in a multi-actor environment } \\
\text { and affect large parts of the population. } \\
\text { Relevance }\end{array}$ \\
& $\begin{array}{l}\text { The rationales for priority-setting decisions should aim to provide a reasonable explanation of how organisations and health } \\
\text { providers seek to meet the health needs of a population under reasonable resource constraints. Specifically, a rationale will be } \\
\text { disposed to finding mutually justifiable terms of cooperation. By 'fair-minded' people, we do not simply mean our friends or }\end{array}$ \\
Appeals and & people who just happen to agree with us. \\
revision & There must be mechanisms for challenge and dispute resolutions regarding priority-setting decisions, and, more broadly, \\
Enforcement & opportunities for revision and improvement of policies in the light of new evidence or arguments. \\
& There must either be a voluntary or public regulation of the process to ensure the first three conditions are met.
\end{tabular}


systems as a whole, then it should be possible to illustrate what such accountability would mean in practical terms... - [17].

This is the first study to apply the AFR framework to evaluate priority-setting decisions that involve selection of drugs in Tanzania. The selection process for drugs to be listed on the national essential medicine list also involves priority setting. We argue that evaluation of these decisions against the AFR framework is one method of improving the process of drug selection. This study therefore reports on the evaluation of the prioritysetting decision for the implementation of artemisininbased combination therapy policy against the four conditions of the AFR framework.

\section{Methods}

\section{Study type and participants}

This was a qualitative study in which in-depth interviews were held with key informants. At least two participants were selected from the following list of institutions: Muhimbili National Hospital (MNH), National Malaria Control Programme (NMCP), WHO office in Tanzania, Ministry of Health $(\mathrm{MoH})$, Tanzania Food \& Drug Authority (TFDA), Medical Stores Department (MSD), and Muhimbili University of Health and Allied Sciences (MUHAS). Participants were experts on malaria research, administration of public health institutions, management of patients, and regulation and management of pharmaceuticals.

\section{Sampling and sample size estimation}

The purposive sampling method was used to select the key informants from the acknowledgements list of the treatment guideline. A sample of 15 participants was selected based on Guest et al.'s recommendations, which imply that, in a piece of research that is narrow in scope and has a homogenous sample, data saturation is achieved after 12 interviews when thematic analysis is employed [23].

\section{Data collection}

Data were collected by in-depth interviews held with key informants using pretested interview guides and reviews of the treatment guideline between May and July 2010. A digital audio recorder was used to record the conversations. The in-depth interview guide consisted of questions corresponding to the AFR framework [17]. Each interview lasted for about one hour. The four conditions of the framework were the main themes. The accessibility of the decision and its rationales were categorised under publicity. Rationales and stakeholder involvement were categorised under relevance. Efficacy, affordability, availability, and cost effectiveness were subcategorised under rationales. Representation, existence of explicit procedures for stakeholder involvement in the task force committee, and consultations were subcategorised under stakeholder involvement.

\section{Data management and analysis}

Interviews were transcribed and then analysed using the ATLAS.ti (ATLAS.ti Scientific Software Development $\mathrm{GmBH}$, Berlin, Germany), a qualitative data analysis (QDA) software, using an editing organising-style method [24]. First the template was prepared based on the interview guide, according to Kvåle's analysis approach [25]. This approach involves the defining of codes before an in-depth analysis of the data. Meaningful units or segments of text in the transcripts and the treatment guideline that correspond to the template or related to this study were identified. Networks for each code with their corresponding quotations were formulated using the QDA program. These networks enabled the researchers to analyse the descriptions of each participant for each code for the transcribed data. Thereafter, memos were written to combine these descriptions with those that emerged from similar codes in the treatment guideline. A network of all codes and their corresponding memos under their subcategories was made, and this enabled the researchers to analyse the descriptions in the memos and reach a conclusion for each subcategory. Finally, a network of the main themes of fairness with their descriptions was made for final analysis and evaluation, in order to determine whether or not the four conditions were satisfied.

\section{Data validity}

To ensure the validity of the data, participants were assured of the aim of the study and their names were anonymised. The in-depth interviews were held in places where interviewees felt comfortable, and the conversations were recorded by using a digital audio recorder. Interviews were transcribed by the interviewer shortly after each session. Telephone numbers of the interviewees were taken so that if any information was missing, they could be called for clarification. Transcripts were read and re-read several times so that the researcher could become familiarised with their contents before analysis. Coding of both the transcripts and the treatment guideline were done separately, and the emerging descriptions were triangulated during memo writing.

\section{Ethical issues}

Ethical clearance (Ref. No. MU/PGS/SAEC/Vol. III/185) was provided by the MUHAS Ethical Review Committee. Participants were asked for verbal informed consent and for permission to be recorded prior to the interviews. 


\section{Results}

Publicity condition

\section{Accessibility of the decision and its rationales}

A majority of the participants reported that the decision about the selection and use of ALu was disseminated to the public by the use of radio, television, and newspaper channels in the national language, Swahili. Meetings with other stakeholders, such as pharmacists, were also held as a means of disseminating this information. They also reported that all the public healthcare facilities from the dispensary to the regional hospital level received the treatment guidelines, which contained the decision and rationales for the prioritisation of ALu.

\section{Publicity condition: evaluation}

The decision about the selection of $\mathrm{ALu}$, but not the reasons behind it, was communicated through advocacy campaigns by National Malaria Control Program (NMCP) and Ministry of Health $(\mathrm{MoH})$ using different media channels in Swahili, the national language. The rationales were written in the treatment guidelines, which are not easily accessible to the public, patients, or other stakeholders who were not directly involved with the task force [26].

\section{Relevance condition Rationales}

Efficacy Participants reported that the problem of parasite resistance to sulphadoxine-pyrimethamine was the major factor that caused the change of the National Malaria Treatment Policy. At the time of this policy change, three choices of artemisinin-based combination drugs recommended by WHO for management of uncomplicated malaria were available. These drugs included sulphadoxine/pyrimethamine-artesunate (SP-AS), amodiaqine-artesunate (AQ-AS), and ALu. ALu had the following advantages over the other drugs: (1) it was the only fixed combination drug among the three, (2) neither of its components had documented reports of parasite resistance, (3) it was safer, as amodiaquine's safety profile had already generated concern among healthcare providers and the general public and (4) it was the most efficacious drug. Affordability

Participants reported that affordability was not discussed because of the agreement between the government and the Global Fund to provide the drug at a subsidized price at the public and faith-based healthcare facilities. This was facilitated by the agreement between WHO and the company that manufactures ALu, which made the drug available at cost price for use in the public sector for malaria-endemic developing countries. However, some participants raised concerns about the sustainability of this financing approach.
... as a country you cannot just sit and wait for somebody to carry the burden of maintaining your population's health, imagine if Global Fund does not keep on sustaining this program!-Representative from MSD

Availability of subsidized artemether-lumefantrine Participants pointed out that, based on Global Fund requirements, the subsidized ALu could only be made available at public and faith-based primary to tertiary healthcare facilities. One participant who works at a tertiary hospital expressed concerns about the low availability of ALu in the primary healthcare facilities:

I receive patients from the dispensaries who have been given sulphadoxine-pyrimethamine and amodiaquine and they sometimes ask why they are still prescribing these drugs when the first-line drug is artemether-lumefantrine!-Physician from $\mathrm{MNH}$

One participant was concerned about the urban-poor populations who have to make a choice between long waiting times and unreliable public health services versus reliable but unaffordable services in the private sector. Participants from National Malaria Control Program (NMCP) said that other strategies were being considered to make sure ALu was also available at a subsidized price in the private-for-profit healthcare facilities.

Effectiveness and cost-effectiveness Participants said that fixed-combination antimalarial drugs have better compliance, hence are more effective compared to nonfixed combinations. However, artemether-lumefantrine's six-dose regimen of four tablets to be taken twice a day with a fatty meal for three successive days was a major concern.

There are obvious improvements, admissions due to severe malaria nowadays are few and this could be explained by the effectiveness of artemether-lumefantrine. As a tertiary hospital we used to have a lot of patients coming in coma due to cerebral malaria; this is not research data but my own observation.-Physician from $\mathrm{MNH}$

About its cost-effectiveness, the majority of participants reported that there were no research data at the time of the policy change that indicated the cost -effectiveness of ALu.

\section{Stakeholder involvement}

Representation and existence of explicit procedures for stakeholder involvement The task force committee was composed of 25 members; 21 were medical doctors and four were pharmacists. The medical doctors were paediatricians, obstetricians/gynaecologists, parasitologists, and pharmacologists from Muhimbili National Hospital (MNH) and Muhimbili University of Health and Allied Sciences (MUHAS). Others were working with the WHO country office, National Malaria Control Program (NMCP) and Integrated Management of 
Childhood Illnesses (IMCI) programs within Ministry of Health $(\mathrm{MoH})$. The pharmacists were working with Medical Store Department (MSD) in the procurement and distribution of drugs, Tanzania Food and Drug Authority (TFDA) in food and drug regulations, and the pharmaceutical services unit at the Ministry of Health. All the committee members were from institutions based in the city of Dar es Salaam. Participants from NMCP who coordinated the policy-change process reported that they did not have written documents on procedures to select the committee members but that they 'knew' the main stakeholders. This approach was criticised by one of the participants:

Sometimes I feel as if it is not adequate because if I had not contacted those people with my findings, I might not have been invited. So there might be other people who are in the field but because they had not made contact with Ministry of Health officials, they did not get the opportunity to be part of the task force committee.-Researcher from MUHAS

Participants reported that patients and the public were indirectly involved through representation and consultations. It was also argued that the task force committee members were potential malaria patients, and therefore, they were themselves representatives of patients and the public. The idea of direct patient and general public involvement in the committee was opposed by some participants who labelled it as inappropriate because policy making is an expert's field.

Consultations Participants reported that many consultations were held before and even after the assembly of the task force committee to get input and technical advice about policy change. Participants from National Malaria Control Program (NMCP) said that the first draft of the guideline was reviewed by experts from the National Institute for Medical Research, Malaria Consortium-Kampala, WHO Afro, Médecins sans Frontières, Ifakara Health Institute, and Eduardo Mondlane-Maputo (Mozambique). They also reported that the draft guidelines were shared by all district and regional medical officers as well as partner countries for technical advice. Councils gave input during the zonal training sessions that were conducted countrywide.

\section{Relevance condition: evaluation Rationales}

The reasons for the prioritisation of ALu appeal to stakeholders because they were based on scientific evidence of clinical efficacy, safety, and formulation profiles. Financing mechanisms were already in place to ensure that the drug would be available and affordable at the public and faith-based healthcare facilities.

\section{Stakeholder involvement}

The study found that there were no transparent procedures to engage the stakeholders. Some stakeholders were involved because of their positions in the relevant institutions, while others were involved through informal links with officials in the Ministry of Health $(\mathrm{MoH})$ or National Malaria Control Program (NMCP). The committee lacked professional, institutional, and countrywide representation. It was largely made up of medical doctors and a few pharmacists; neither nurses nor medical lab personnel were included. All the physicians, specialists, and academicians within the task force committee came from one hospital, i.e. Muhimbili National Hospital (MNH) and one university, Muhimbili University of Health and Allied Sciences (MUHAS). There were other similar institutions in the country that could have provided members to increase representation.

\section{Appeals and revision condition}

Participants said that it is not easy for anybody to appeal against the policy; however, if established facts and evidence on toxicities and level of resistance were to be provided, the policy decision could be revised.

I think the best way for people to appeal is to provide scientific data in the scientific forum; it is impossible for an individual to come up and say this is not the right drug. From the community side, I think the only mechanism they have is to express their concerns in the newspapers and other media; otherwise, I don't see any other channels at the moment.-Participant from TFDA

Pharmacovigilance forms, which are used as post-marketing surveillance tools in monitoring drug safety, were mentioned as effective tools to generate evidence on the safety profile of ALu. However, one participant disagreed with the reliability of this approach.

Most of our clinics are crowded, before you can take a minute or two to fill in the pharmacovigilance form, 20 people are already shouting at you, so it is one avenue but is not satisfactory.-Physician from $\mathrm{MNH}$

\section{Appeals and revision condition: evaluation}

There is no mechanism in place to appeal against the decision to prioritiseALu.

\section{Enforcement condition}

There was no enforcement mechanism. The demand for enforcement was seen as new and was considered unnecessary by some participants. Other participants said that an independent enforcement body is necessary during a treatment policy change.

I am not sure if there was a watchdog. I think it is really very important because sometimes people say the 
drug companies are pushing for policy change.-Pharmacologist from MUHAS

\section{Enforcement condition: evaluation}

Despite growing concerns about the influence of pharmaceutical companies on change of treatment policies, we did not find any enforcement mechanism to ensure the other three conditions were met.

\section{Discussion}

This study evaluated the process of changing the Tanzania National Malaria Treatment Policy against the AFR framework. The process resembled other priority-setting decisions, in that it involved making choices among competing alternatives in the context of limited resources, conflicting evidence, diverse stakeholder involvement, and the vagaries of political cycles [27]. The influence of power was evident insofar as some individuals, groups, and institutions were better positioned than others to influence decision outcomes. Power differences were associated with professional and institutional status, the reliability of research findings, ownership of financial resources, and the historical dominance of the public healthcare delivery system.

When evaluated against the AFR, this study found that the publicity condition was not fully satisfied. The study found that the decision to prioritiseALu was made public, but not the reasons behind it. Publicity requires that the decision and its rationales, such as the ones for coverage of new drugs like ALu, be publicly accessible to all stakeholders, including the patients [17]. Similar findings have been reported at the district level, where again the decision but not the reasons behind it were communicated to stakeholders [21].

The study found that the rationale but not the stakeholder involvement part of the relevance condition was satisfied. ALu was the only artemisinin-based antimalarial drug that was in a fixed-combination formulation. It was also the most efficacious drug and neither of its two components had any documented reports of parasite resistance [6]. Cost-effectiveness evidence was not used to inform the decision, but studies have shown that ALu was the most cost-effective drug among the available alternatives $[28,29]$. The selection of ALu was influenced by an agreement between the WHO and Novartis, the company that manufactures ALu, to monitor the global demand and supply of the drug. In exchange, this agreement enabled malaria-endemic developing countries to procure the drug at cost price for distribution in the public healthcare facilities [2]. However, the major concern among the stakeholders is the sustainability of the existing financing arrangement. Affordable Medicines Facility-Malaria (AMFm) is currently pioneering a pilot study to involve the private for-profit facilities [30] because it has been shown that the private sector plays a major role in malaria case management [31,32]. These attempts to increase access to artemisinin-based antimalarials must ensure that the delivery, financial, and governance arrangements are compatible with the national context needs [33]. Although artemisinin-based antimalarial drugs are the best hope for malaria case management, there are various challenges facing their use. In areas where ALu has been deployed, patients frequently do not adhere to its six-dose regimen, hence compromising its effectiveness [34]. Studies have also shown that many doses of these expensive drugs are lost due to symptomatic diagnosis and inadequate slide reading [35-37].

The study found that stakeholders were not adequately involved and represented. Appropriately conducted stakeholder participation not only facilitates and widens discussions in the decision-making process but also enhances publicity [17]. The task force committee was also not representative. Proper representation of healthcare professionals who also represent different institutions at the discussion table was necessary. This would have complemented the findings of the IMPACT Project, which was carried out in order to inform the policy change decision [38], which ultimately would have enhanced accountability for reasonableness.

Even though we argue in favour of wide professional and institutional representation, this should not aim to replace patients and the general public. There is evidence that health professionals' views can differ considerably from those of their patients [39]. However, the involvement of the public and patients in an area populated by professionals is a challenge, especially in developing countries. It has been shown that potential stakeholders at the district level in Tanzania have no knowledge, skills, and experience to be able to participate effectively in priority-setting decisions [20]. Use of large-scale surveys and the rapid appraisal method have been tested and have proved successful in developing countries. The views of key groups of people, like teachers, shop owners, and students, elicited through interviews can adequately represent the opinions of their communities [40].

The appeals and revision condition was not satisfied. To our knowledge, there are no formal appeals mechanisms in the Tanzanian healthcare system for policy decisions relating to access to new drugs or healthcare. Pharmacovigilance systems in developing countries are weak, and hence cannot be considered an efficient appeal mechanism to inform policy makers of a drug causing harm to its users. The lack of reliable appeal mechanisms suggest that good arguments and evidences against the original decision and which aim at improving faulty policy decisions can have no clear way back 
onto the decision table. A study by Maluka et al. reported a lack of clear formal channels for appeals at the district level in Tanzania [21].

The enforcement condition was not satisfied. This condition recognises that public or private regulation may be necessary if self-regulation proves unsuccessful or inadequate. There are growing concerns about the influence of pharmaceutical companies, which lobby and push for national treatment policy changes, especially in developing countries $[14,16]$. Hence, the establishment of an independent regulatory body to guarantee fair process during policy change is necessary. Other studies in Tanzania have also reported lack of enforcement mechanisms for priority-setting decisions due to poor organisation, unclear lines of authority, low level of public awareness, and inadequate public enforcement mechanisms $[21,22]$.

\section{Strengths and limitations of the study}

The qualitative research method is the best approach to study complex phenomena when a researcher wants to capture the views of individual persons, including their feelings, understandings, interpretations, beliefs, and ideologies. Through the use of this method, the participants shared their stories and conflicting views beyond what the researchers expected to hear or what is available in the literature.

The study used the AFR as an evaluation framework; however, there are objections to its use as an approach to achieve justice and fairness [41,42]. Moreover, differences may exist between the AFR's definition and participants' views on the concept of fairness and its evaluation. The results obtained from the interviewed participants may not be the general views of the whole group, as we interviewed only a part of the task force team who may not be the true representation of the group. As emerged from the interviews, some decisions were made before the assembly of the task force committee, while other decisions were not made by the task force committee per se. Due to resource and time constraints, we did not interview any individuals from the consultation group, the general population, or patients' representatives. Other stakeholders include healthcare professionals outside the task force committee, the ALu manufacturing company, and Global Fund as the financing institution. We strongly believe that this study is missing the views of other individuals who may possess important and reliable information related to this study.

\section{Conclusions}

This study applied the AFR framework to evaluate the priority-setting decision for new antimalarial drugs at the national level. The study found that the priority- setting decision in favour of ALu failed to satisfy the four conditions of fair process prescribed in the ethical framework of accountability for reasonableness. The findings of this study are consistent with the reports of other studies that evaluated priority-setting decisions against AFR at the district level. The study emphasises the need to involve a wide range of stakeholders in priority-setting decisions. It further highlights the need to make priority-setting decisions and their rationales public and to establish mechanisms for appeal, revision, and regulation in order to enhance fairness and legitimacy of the decision outcomes.

\section{Acknowledgements}

We thank Norad's Masters Programme, NOMA, which funded ATM to carry out this study. We also thank all the participants for consenting to participate in the study.

\section{Author details}

${ }^{1}$ Department of Pharmaceutics and Pharmacy Practice, School of Pharmacy, Muhimbili University of Health and Allied Sciences, Dar es Salaam, Tanzania. ${ }^{2}$ Centre for International Health, University of Bergen, Bergen, Norway. ${ }^{3}$ Department of Medicinal Chemistry, School of Pharmacy, Muhimbili University of Health and Allied Sciences, Dar es Salaam, Tanzania.

\section{Authors' contributions}

ATM conceived and designed the study, collected the data, performed the analysis, and prepared the draft of the manuscript. EAK fine-tuned the study design and coordinated the data collection and analysis. All authors read and approved the final manuscript.

\section{Competing interests}

The authors declare that they have no competing interests.

Received: 15 July 2011 Accepted: 18 March 2012

Published: 18 March 2012

\section{References}

1. Mutabingwa TK: Artemisinin-based combination therapies (ACTs): best hope for malaria treatment but inaccessible to the needy! Acta Trop 2005, 95:305-315.

2. WHO: World Malaria Report, 2009 Geneva, Switzerland: World Health Organization; 2009

3. Vuorenkoski L, Toiviainen $H$, Hemminki E: Decision-making in priority setting for medicines-A review of empirical studies. Health Policy 2008, 86:1-9.

4. Martin DK, Hollenberg D, MacRae S, Madden S, Singer P: Priority setting in a hospital drug formulary: a qualitative case study and evaluation. Health Policy 2003, 66:295-303.

5. WHO: Antimalarial drug combination therapy: Report of a technical consultation Geneva, Switzerland: World Health Organization; 2001.

6. National Malaria Control Programme: National Guidelines for Malaria Diagnosis and Treatment Malaria control Series 11; Dar es Salaam, Tanzania; 2006.

7. Canadian Agency for Drugs and Technologies in Health: Guidelines for the economic evaluation of health technologies: Canada. 3 edition. Ottawa: Canadian Agency for Drugs and Technologies in Health; 2006.

8. Ministry of Finance and Economic Affairs: The Economic Survey. 2010 Dar es Salaam: KIUTA; 2009, 226

9. Ministry of Health: Second Health Sector Strategic Plan (2003-2008): Reforms towards delivering quality health services and clients satisfaction Dar es Salaam-Tanzania; 2003.

10. Ministry of Health: Proposals for Health Sector Reforms Dar es SalaamTanzania; 1994.

11. Humba E: Pioneering Social Health Insurance in Tanzania: The case of National Health Insurance Fund (NHIF). Proceedings of Swiss TPH Spring 
Symposium on Improving Access Through Effective Financing Basel: UBS Training and Conference Centre; 2011.

12. Mtei GP, Mulligan J, Palmer N, Kamuzora P, Ally M, Mills A: An assessment of the health financing system in Tanzania: implications for equity and social health insurance. Report on Shield Work Package 1, Dar es Salaam, Tanzania 2007.

13. Kramera AR, Dickinson KL, Anderson RM, Fowler VG, Miranda ML, Mutero CM, Saterson KA, Wiener JB: Using decision analysis to improve malaria control policy making. Health Policy 2009, 92:133-140.

14. Amin AA, Zurovac D, Kangwana BB, Greenfield J, Otieno DN, Akhwale WS, Snow RW: The challenges of changing national malaria drug policy to artemisinin-based combinations in Kenya. Malar J 2007, 6(72).

15. Mubyazi GM, Miguel A, Gonzalez B: Research influence on antimalarial drug policy change in Tanzania: case study of replacing chloroquine with sulfadoxine-pyrimethamine as the first-line drug. Malar J 2005, $4(51)$.

16. Holly AN, Durrheim D, Rima S: The process of changing national malaria treatment policy: lessons from country-level studies. Health Policy Plan 2003, 19(6):356-370.

17. Daniels N, Sabin J: Setting limits fairly: Can we learn to share medical resources? Oxford: Oxford University Press; 2002.

18. Daniels N, Sabin JE: Limits to health care: fair procedures, democratic deliberation and the legitimacy problem for insurers. Philos and Public Aff 1997, 26:303-350.

19. Daniels N, Sabin J: Just health: meeting health needs fairly New York: Cambridge University Press; 2008.

20. Mshana S, Shemilu H, Ndawi B, Momburi R, Olsen OE, Byskov J, Martin DK: What do District Health Planners in Tanzania think about improving priority setting using 'Accountability for Reasonableness'? BMC Health Serv Res 2007, 7(180)

21. Maluka S, Kamuzora P, Miguel S, Byskov J, Olsen E, Shayo E, Ndawi B, Hurtig A: Decentralized health care priority-setting in Tanzania: evaluating against the accountability for reasonableness framework. SocSci Med 2010, 71:751-759.

22. Maluka S, Kamuzora P, Miguel S, Byskov J, Olsen E, Shayo E, Ndawi B, Hurtig A: Implementing accountability for reasonableness framework at district level in Tanzania: a realist evaluation. Implementation Sci 2011, 6(11)

23. Guest $\mathrm{G}$, Bunce A, Johnson L: 'How many interviews are enough? An experiment with data saturation and variability. Field Methods 2006, 18:59-82.

24. Miller W, Crabtree B: Clinical research: a multimethod typology and qualitative roadmap. In Doing qualitative research.. 2 edition. Edited by: Crabtree B, Miller W. London: Sage; 1999:20-24.

25. Kvale S: Interviews, An Introduction to Qualitative Research Interviewing Thousand oaks: Sage publications; 1999.

26. Minzi OMS, Haule AF: Poor knowledge on new malaria treatment guidelines among drug dispensers in private pharmacies in Tanzania: the need for involving the private sector in policy preparation and implementation. East Afr J Public Health 2008, 5(2):117-121.

27. Gibson JL, Martin DK, Singer PA: Setting priorities in health care organizations: Criteria, processes and parameters of success. BMC Health Serv Res 2004, 4(25).

28. Wiseman V, Kim M, Mutabingwa TK, Whitty CJM: Cost-effectiveness study of three antimalarial drug combinations in Tanzania. PLoS Medicine 2006, $3(10)$

29. Chanda P, Masiye F, Chitah B, Sipilanyambe N, Hawela M, Banda P, Okorosobo T: A cost-effectiveness analysis of artemetherlumefantrine for treatment of uncomplicated malaria in Zambia. Malar J 2007, 6(21).

30. Matowe L, Adeyi $\mathrm{O}$ : The quest for universal access to effective malaria treatment: how can the AMFm contribute? Malar J 2010, 9(274).

31. Jowett M, Miller $\mathrm{NJ}$ : The financial burden of malaria in Tanzania: implications for future government policy. Int I Health Plann Manage 2005, 20:67-84.

32. Hanson K, Goodman C, Lines J, Meek S, Bradley D, Mills A: The Economics of Malaria Control Interventions. Global Forum for Health Research. World Health Organization 2004

33. Nabudere $H$, Upunda GL, Juma M: Policy brief on improving access to artemisinin-based combination therapies for malaria in the East African community. Int J Technol Assess Health Care 2010, 26(2):255-259.
34. Lemma H, Lofgren C, San Sebastian M: Adherence to a six-dose regimen of artemether-lumefantrine among uncomplicated Plasmodium falciparum patients in the Tigray Region, Ethiopia. Malar J 2011, 10(349).

35. Reyburn H, Mbakilwa H, Mwangi R, Mwerinde O, Olomi R, Drakeley C, Whitty CJM: Rapid diagnostic tests compared with malaria microscopy for guiding outpatient treatment of febrile illness in Tanzania: randomised trial. BMJ 2007, 334(403)

36. Reyburn H, Mbatia R, Drakeley C, Carneiro I, Mwakasungula E: Over diagnosis of malaria in patients with severe febrile illness in Tanzania: a prospective study. BMJ 2004, 329:1212-1218.

37. Mwanziva C, Shekalaghe S, Ndaro A, Mengerink B, Megiroo S, Mosha F, Sauerwein R, Drakeley C, Gosling R, Bousema T: Overuse of artemisinincombination therapy in MtowaMbu (river of mosquitoes), an area misinterpreted as high endemic for malaria. Malar J 2008, 7(232).

38. IHRDC: IMPACT Tanzania, Interdisciplinary Monitoring Program for Antimalarial Combination Therapy in Tanzania.[http://www. mimcom. org. uk/ifakara/impact. htm].

39. Bowling A, Jacobson B, Southgate $L$ : Exploration in consultation of the public and health professionals on priority setting in an inner London health district. SocSci Med 1993, 37(7):851-857.

40. Mullen PM: Public involvement in health care priority setting: an overview of methods for eliciting values. Health Expect 1999, 2:222-234.

41. Rid A: Justice and procedure: how does "accountability for reasonableness" result in fair limit-setting decisions? J Med Ethics 2009, 35:12-16.

42. Friedman A: Beyond accountability for reasonableness. Bioethics 2008 , 22(2):101-112.

doi:10.1186/1748-5908-7-18

Cite this article as: Mori and Kaale: Priority setting for the implementation of artemisinin-based combination therapy policy in Tanzania: evaluation against the accountability for reasonableness framework. Implementation Science 2012 7:18.

\section{Submit your next manuscript to BioMed Central and take full advantage of:}

- Convenient online submission

- Thorough peer review

- No space constraints or color figure charges

- Immediate publication on acceptance

- Inclusion in PubMed, CAS, Scopus and Google Scholar

- Research which is freely available for redistribution 